\title{
Rhabdomyolysis in 119 students after repetitive exercise
}

\author{
A C-M Lin, C-M Lin, T-L Wang, J-G Leu
}

Br J Sports Med 2005;39:e3 (http://www.bisportmed.com/cgi/content/full/39/1/e3). doi: 10.1136/bjsm.2004.013235

Exercise induced rhabdomyolysis is well known, but has rarely been reported in high school students. This is the report of 119 cases in high school students who exercised vigorously (120 push ups in five minutes) in cold weather. Most of them developed muscle pain and dark urine within two to four days of the exercise.

$\mathrm{E}$ xercise induced rhabdomyolysis has been well described. ${ }^{1}$ Rhabdomyolysis may be accompanied by increased serum creatine phosphokinase (CPK) activity and myoglobinuria. It has many potential causes. However, its association with repetitive exercise in a high school has rarely been reported. We describe exercise induced rhabdomyolysis in 119 high school students who exercised vigorously in cold weather $\left(12^{\circ} \mathrm{C}\right)$ in Taipei. Each student had been told to perform 120 push ups within five minutes by their gymnastics teacher as a test. Most of them developed muscle pain and dark urine two to four days after the exercise.

The students presented to our emergency department after the onset of dark urine and generalised muscle aches. There were 74 male and 45 female patients (age range 17-18 years (mean 17.6)). Serum CPK activity was 55-174 260 U/l (mean $36512 \mathrm{U} / \mathrm{l}$ ) (normal range 50-450 U/1); 89 students had abnormal levels. All urinary myoglobin tests were negative. Serum blood urea nitrogen and creatinine were normal. No cases of acute renal failure were identified. Most of the students were discharged after adequate oral hydration and rest. Twenty required hospital admission because of poor oral hydration and severe muscle pain. They were later discharged without organ damage.

\section{DISCUSSION}

Rhabdomyolysis is a clinical syndrome in which injury to muscle cells is such that their contents, including myoglobin and creatine kinase, leak into the vascular compartment, resulting in acute tubular necrosis or acute renal failure. A healthy person can develop rhabdomyolysis after heavy, prolonged, exhaustive, or repetitive exercise. The patient presents with classic signs of rhabdomyolysis. A definitive diagnosis is made by laboratory tests including serum CPK and urine myoglobin. In patients with CPK activity in excess of five times the normal who also have associated risk factors, the diagnosis is probably rhabdomyolysis. ${ }^{23}$ Myoglobin is difficult to assay in serum and is only transiently found in urine, so serial CPK measurements are used to monitor the patient's progress. Myoglobin will not always be present even though the patient has tea coloured urine because it may disappear from the plasma within six hours of injury. The hallmark signs of rhabdomyolysis are muscle weakness, muscle pains, and dark urine. Common causes are immobilisation, cocaine, methamphetamine, heroin, seizure, trauma, and excessive exercise. The most common are drug related. ${ }^{4}$ In this case, rhabdomyolysis occurred because of fasting, probable hypothermia, and vigorous muscular activity related to the repetitive exercise. The more strenuous or prolonged the exercise, the more damage is incurred. The incidence of rhabdomyolysis is lower in women than men. ${ }^{5}$ Rhabdomyolysis involves injury to the skeletal muscles leading to leakage of potentially toxic cellular contents into the systemic circulation. The most important complication is acute renal failure, which occurs in $5-7 \%$ of cases. ${ }^{6}$ Prevention of myoglobinuric acute renal failure involves maintenance of circulating blood volume by adequate fluid replacement, alkalinisation of the urine, and maintenance loop diuretics. Aggressive intravenous or adequate oral hydration during the first $24-72$ hours is the optimum treatment for rhabdomyolysis.

Fortunately, the emergency doctor in this case recognised the symptoms of rhabdomyolysis early and sought diagnosis and treatment. All the students were sent to the emergency department for screening as they had muscle pain and dark urine. This quick action probably prevented acute renal failure and possibly a more serious outcome. All the patients were previously healthy. This case should serve as a warning that repetitive exercise can cause rhabdomyolysis. Prospective exercise and gymnastics teachers need to know about the potentially adverse effects of repetitive exercise.

\section{Authors' affiliations}

A C-M Lin, C-M Lin, T-L Wang, Emergency Department, Shin Kong Wu Ho-Su Memorial Hospital, Taipei, Taiwan

J-G Leu, Division of Nephrology Medicine, Shin Kong Wu Ho-Su Memorial Hospital

Correspondence to: Dr Leu, Emergency Department, Shin Kong Wu HoSu Memorial Hospital, Taipei, Taiwan; m002001@ms.skh.org.tw

Accepted 15 June 2004

Conflict of interests: none declared

\section{REFERENCES}

1 Sinert R, Kohl L, Rainone T, et al. Exercise-induced rhabdomyolysis. Ann Emerg Med 1994;23:1301-6.

2 Poels PJE. Rhabdomyolysis: a review of the literature. Clin Neurol Neurosurg 1993;95:175-92.

3 Walsworth $M$, Kessler T. Diagnosing exertional rhabdomyolysis: a brief review and report of two cases. Mil Med 2001;166:275-7.

4 Chin RL, Sporer KA, Rimpel NR. Clinical characteristics and outcomes of rhabdomyolysis: a case review of 252 patients. Ann Emerg Med 1999;34:4

5 Rinard J, Clarkson PM, Smith LL, et al. Response of males and females to highforce eccentric exercise. J Sports Sci 2000;18:229-36.

6 Soni SN, McDonald E, Marino C. Rhabdomyolysis after exercise. Postgrad Med 1993;94:128-32. 